\title{
PRECISE MEASUREMENTS OF THE COSMIC RAY ANTIPROTON SPECTRUM WITH BESS INCLUDING THE EFFECTS OF SOLAR MODULATION
}

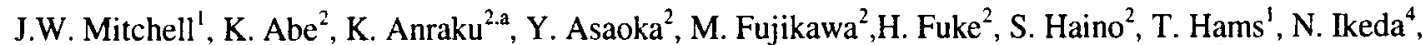

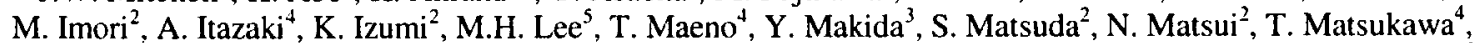 \\ H. Matsumoto ${ }^{2}$, H. Matsunaga ${ }^{2 . b}$, T. Mitsui ${ }^{4 . c}$, A. Moiseev ${ }^{1}$, M. Motoki $^{2 . c}$, J. Nishimura $^{2}$, M. Nozaki ${ }^{4}$, H. Omiya ${ }^{3}$, \\ S. Orito ${ }^{2 . d}$, J.F. Ormes', T. Saeki ${ }^{2}$, T. Sanuki ${ }^{2}$, M. Sasaki', E.S. Seo ${ }^{5}$, Y. Shikaze ${ }^{4}$, T. Sonoda ${ }^{2}$, S.A. Stephens', \\ R.E. Streitmatter', J. Suzuki ${ }^{3}$, Y. Takasugi ${ }^{4}$, K. Tanaka ${ }^{3}$, K. Tanizaki ${ }^{4}$, I. Ueda $^{2}$, J.Z. Wang, Y. Yajima ${ }^{6}$,

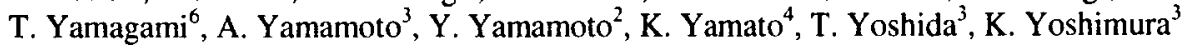 \\ 'NASA Goddard Space Flight Center, Greenbelt, Maryland 20771, USA \\ ${ }^{2}$ The University of Tokyo, Bunkyo, Tokyo 113-0033, Japan \\ ${ }^{3}$ High Energy Accelerator Research Orgnization (KEK), Tuskuba, lbaraki 305-0801, Japan \\ ${ }^{4}$ Kobe University, Kobe, Hyogo 657-8501, Japan \\ ${ }^{5}$ The University of Maryland, College Park, Maryland 20742, USA \\ ${ }^{6}$ Institute of Space and Astronautical Science (ISAS), Sagamihara, Kanagawa 229-8510, Japan
}

\begin{abstract}
The Balloon Borne Experiment with a Superconducting Spectrometer (BESS) has measured the energy spectrum of cosmic-ray antiprotons between 0.18 and $4.20 \mathrm{GeV}$ in eight flights between 1993 and 2002. Above about 1 $\mathrm{GeV}$, models in which antiprotons are secondary products of the interactions of primary cosmic rays with the interstellar gas agree with the BESS antiproton spectrum. Below $1 \mathrm{GeV}$, the data show a possible excess antiproton flux compared to secondary model predictions, suggesting the presence of an additional source of antiprotons. The antiproton/proton ratios measured between 1993 and 1999, during the Sun's positive-polarity phase, are consistent with simple models of solar modulation. However, results from the 2000 flight, following the solar magnetic field reversal, show a sudden increase in the antiproton/proton ratio and tend to favor a charge-signdependent drift model. To extend BESS measurements to lower energies, an evolutionary instrument, BESS-Polar, is under construction for polar flight in 2004.
\end{abstract}

\section{INTRODUCTION}

Measurements of the antiproton component in the cosmic radiation address questions ranging from the origin and transport of cosmic rays to the apparent absence of cosmological antimatter and, perhaps, the nature of the dark matter. Antiprotons are expected as secondary products of the interaction of primary cosmic-ray nuclei (mainly protons) with the interstellar medium (ISM). That this process is the main source of cosmic-ray antiprotons was indicated by the first BESS (Yoshimura et al. 1995; Moiseev et al. 1997) and IMAX (Mitchell et al. 1996) measurements of mass-resolved antiprotons. At $1 \mathrm{GeV}$, the ratio of the antiproton to proton fluxes is about $10^{-5}$. These rare particles must be uniquely identified in the presence of a large flux of cosmic-ray electrons, muons, and nuclei, requiring a careful application of instrumental and analysis techniques.

The low-energy antiproton spectrum in the energy range of 0.18 to $4.2 \mathrm{GeV}$ has been measured with good statistics in subsequent BESS flights (Nishimura et al. 1997, Matsunaga et al. 1998, Orito et al. 2000, Maeno et al. 2001, Asaoka et al. 2002). As summarized in Table 1, these flights, through 2002, total 160 hours of observation time and more than 2100 cosmic ray antiprotons have been identified. The secondary origin of most of the antiprotons is now well established (Orito et al. 2000). This conclusion is supported by the measurements of

\footnotetext{
${ }^{a}$ Currently at Kanagawa University

b Currently at Tsukuba University

c Currently at Tohoku University

d Deceased
} 
IMAX (Mitchell et al. 1996) and CAPRICE94 (Boezio et al. 1997) in energy ranges similar to BESS and by higher energy measurements from MASS91 (Basini et al. 1999), CAPRICE98 (Boezio et al. 2001), and HEAT-pbar (Beach et al. 2001). However, in addition to secondary production, there could be other, more exotic, antiproton sources such as the annihilation of neutralinos or the evaporation of primordial black holes. Because the secondary antiproton spectrum has a sharp peak around $2 \mathrm{GeV}$, antiproton contributions well above or below this peak from other sources could cause an apparent flattening of the spectrum. There are some indications of this flattening in the BESS low-energy data from 1995 and 1997 (Orito et al. 2000).

At low energies, antiproton measurements are strongly affected by the outflowing solar wind. Thus, accurate measurements of the spectra of singly-charged particles, and the effects of solar modulation on those spectra, are critical to understanding the origin of cosmic-ray antiprotons and potential signatures of exotic matter in the measured antiproton spectra. They are equally important as independent scientific goals.

BESS is usually flown from a high-latitude site to access low magnetic rigidity (momentum/charge) particles penetrating the Earth's magnetic field. BESS has been flown from Lynn Lake, Manitoba, Canada, eight times (Anraku et al. 1996; Yamamoto et al. 1998; Maeno et al. 2001) with continuous improvement in the instrument live time and performance. Each flight reached a float altitude of about $35 \mathrm{~km}$ (residual pressure $\sim 5 \mathrm{mbar}$ ). The high-latitude flights through 2002 are summarized in Table 1.

Table 1. Progress of the BESS balloon flights in Canada.

\begin{tabular}{llllllllll}
\hline Year & unit & 93 & 94 & 95 & 97 & 98 & 99 & 2000 & 2002 \\
\hline Float time & [hours] & 17.5 & 17.0 & 19.5 & 20.5 & 22 & 34.5 & 44.5 & 16.5 \\
Observation time & {$[$ hours] } & 14 & 15 & 17.5 & 18.3 & 20.0 & $31.3+\left(2.8^{*}\right)$ & $32.5+\left(2.5^{*}\right)$ & $11.3+\left(2.3^{*}\right)$ \\
Events recorded $\left[10^{6}\right.$ events] & 4.0 & 4.2 & 4.5 & 16.2 & 19.0 & $16.8+\left(2.3^{*}\right)$ & $15.0+\left(2.0^{*}\right)$ & $11.8+\left(1.9^{*}\right)$ \\
Data volume $[\mathrm{GB}]$ & 4.5 & 6.5 & 8.0 & 31 & 38 & 41 & 38 & 56 \\
TOF resolution $(\sigma)[\mathrm{ps}]$ & 300 & 300 & 100 & 75 & 75 & 75 & 75 & 75 \\
Silica-aerogel index & & & & 1.032 & 1.020 & 1.020 & 1.020 & 1.020 \\
Antiprotons observed & 6 & 2 & 43 & 415 & 384 & 668 & 558 & $\mathrm{TBD}$ \\
Antiproton energy [GeV] & $0.18-0.5$ & $0.18 \sim 0.5$ & $0.18 \sim 1.5$ & $0.18 \sim 3.6$ & $0.18 \sim 4.2$ & $0.18 \sim 4.2$ & $0.18 \sim 4.2$ & $0.18 \sim 4.2$ \\
Anti-He/He limit & $2.2 \times 10^{-5}$ & $4.3 \times 10^{-6}$ & $2.4 \times 10^{-6}$ & $1.4 \times 10^{-6}$ & $1.0 \times 10^{-6}$ & $8 \times 10^{-7}$ & $6.8 \times 10^{-7}$ & TBD \\
\hline
\end{tabular}

* (data also taken during balloon ascent)

\section{THE BESS INSTRUMENT}

BESS is a magnetic-rigidity spectrometer with large geometrical acceptance, using a thin superconducting solenoidal magnet and a high-resolution drift-chamber tracking system (Orito 1987, Yamamoto et al. 1994, Ajima et al. 2000). This spectrometer is coupled to a state-of-the-art time-of-flight (TOF) system and a silica-aerogel Cherenkov counter (ACC). In its present configuration, the detectors and magnet are contained in an external pressure vessel maintained at 1 atmosphere and are arranged in a cylindrical configuration with a horizontal axis, as shown in Figure 1. The horizontal configuration enables the spectrometer to be compact and to have a wide opening angle, giving an acceptance of $0.3 \mathrm{~m}^{2} \mathrm{sr}$. The total mass of the instrument is currently $-2,300 \mathrm{~kg}$.

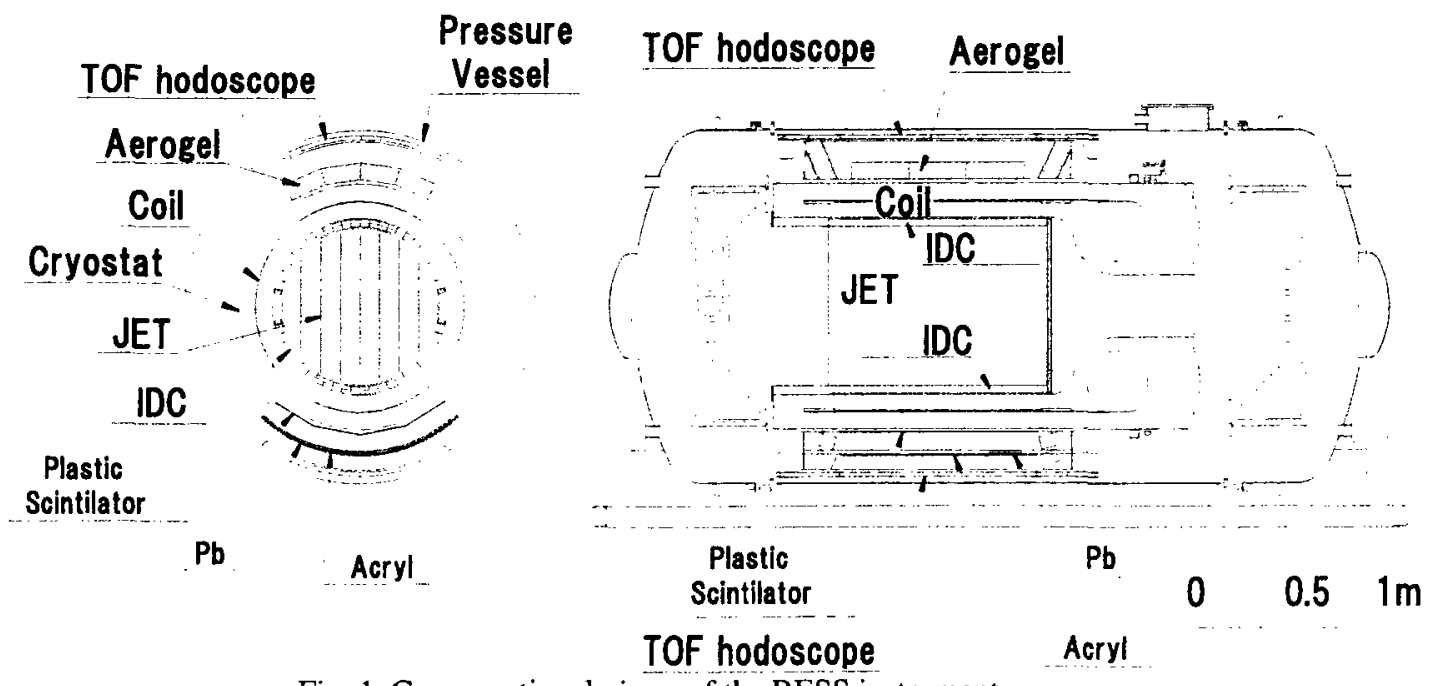

Fig. 1. Cross-sectional views of the BESS instrument. 
The magnetic rigidity and charge-sign of incident particles are determined by measuring track curvature in the 1 Tesla axial field, which is uniform to $15 \%$ (Yamamoto et al. 1988). Curvature is measured using a central JETtype drift chamber (JET), which fills the warm-bore high-field region of the magnet. This chamber has a position resolution of $200 \mu \mathrm{m}$ in the bending plane and provides up to 24 track positions. The most probable maximumdetectable-rigidity (MDR) of the system used for flights through 2000 is $200 \mathrm{GV}$, and $90 \%$ of the events have MDR $>100 \mathrm{GV}$. Axial positions with a resolution of 1.5 $\mathrm{cm}$ for $|Z|>1$ particles are given by charge-division readout on both ends of the JET sense wires. Since the magnetic field is highly uniform inside the solenoid, a very sharp deflection resolution can be realized (Sanuki et al. 2000). This enables the BESS experiment to provide very precise energy resolution and high statistics by allowing the full geometric acceptance to be used at all energies.

Inner drift chambers (IDC) are located just inside the cryostat. In combination with an outer hodoscope provided by a set of outer drift chambers (ODC) through 1995 , and subsequently by the TOF system, they are used on-line by a second-level trigger system to select events rapidly for further processing. The BESS trigger detects
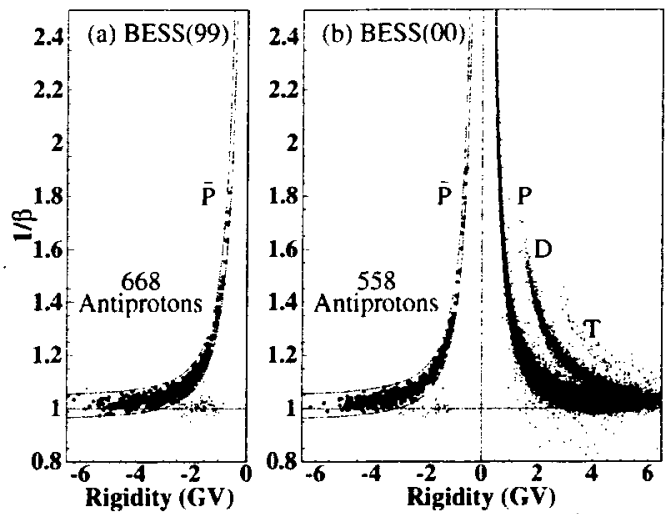

Fig. 2. Separation of antiprotons and hydrogen isotopes in the 1999 and 2000 BESS flights. negatively-charged particles with high efficiency, while sampling a fraction of the much more abundant positively-charged protons and helium. These chambers also provide 4 bending-plane points by drift-time measurement with a precision of $200 \mu \mathrm{m}$, and axial position by vernier-pad readout with $500 \mu \mathrm{m}$ precision.

In successive flights, the TOF measurement of BESS has regularly been improved (Shikaze et al. 2000). Its resolution is now $75 \mathrm{ps}$, which yields a resolution for relativistic particles of $0.008 \mathrm{in} 1 / \beta$. This allows antiprotons to be clearly mass-resolved up to energies of $1.4 \mathrm{GeV}$. Upward-moving and downward-moving particles are cleanly separated with negligible chance of mistaking the direction of motion and consequently the charge-sign.

The aerogel Cherenkov counter (ACC), added in 1997 for rejection of light background particles (muons and electrons), initially used an optical index, $n$, of 1.032 to cover antiproton energies up to about $3.6 \mathrm{GeV}$ (Asaoka et al. 1998). Currently, $n=1.02$ is used. This yields -15 photoelectrons $(Z=1, \beta=1)$ and extends measurements up to $4.2 \mathrm{GeV}$. Figure 2, showing results from the 1999 and 2000 flights, illustrates the separation presently achieved between antiprotons and hydrogen isotopes using the TOF and ACC. A shower counter, using lead plates and acrylic Cherenkov detectors and covering about $20 \%$ of the geometric acceptance, was flown in 1999 and 2000 to improve rejection of electrons for measurements of atmospheric muons (Sanuki et al. 2001a).

For 2001, the rigidity resolution of the spectrometer was greatly improved by the development of new JET, IDC, and ODC chambers. This resulted in a factor of five improvement in the momentum resolution, giving an MDR of 1 TV. Known as BESS-TeV (Sanuki et al. 2001b) this version of the instrument was flown in 2001 from $\mathrm{Ft}$. Sumner, NM, where the geomagnetic cutoff is about $4.3 \mathrm{GV}$, and in 2002 from Lynn Lake.

\section{ANTIPROTON MEASUREMENTS}

Antiprotons are a unique tool to probe the propagation process. From the measured primary

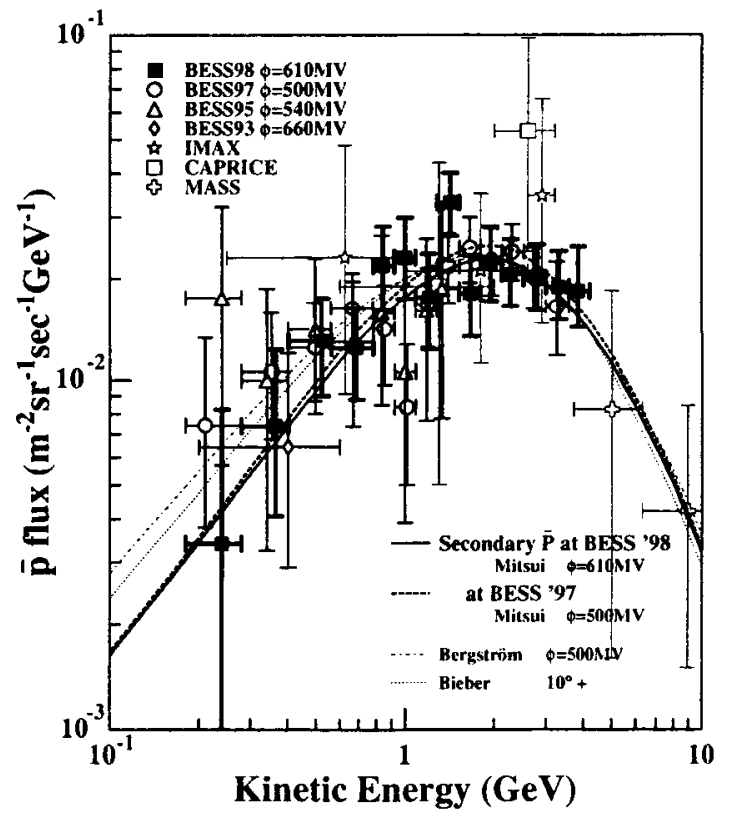

Fig. 3. BESS antiproton spectra (1993-1998) at the top of the atmosphere together with previous data and model calculations of secondary-production spectra. 
cosmic-ray flux and knowledge of the antiproton production mechanism, the flux of secondary antiprotons resulting from the interaction of primary cosmic rays with the ISM can be calculated using models of galactic and heliospheric transport. Figure 3 shows the energy spectrum measured by BESS in comparison with other experiments (Maeno et al. 2001). A clear peak in the energy spectrum at $2 \mathrm{GeV}$, characteristic of secondary production, was first observed in BESS-97 data (Orito et al. 2000) and was confirmed in BESS-98 data (Maeno et al. 2001). Three calculations of the secondary antiproton flux (Mitsui et al. 1996, Bergstroem et al. 1999, Bieber et al. 1999) are shown in Figure 3. These reproduce the observed flux at the peak region around $2 \mathrm{GeV}$ with $10-15 \%$ accuracy. This implies that most of the observed antiprotons are secondary and that the propagation models are basically correct.
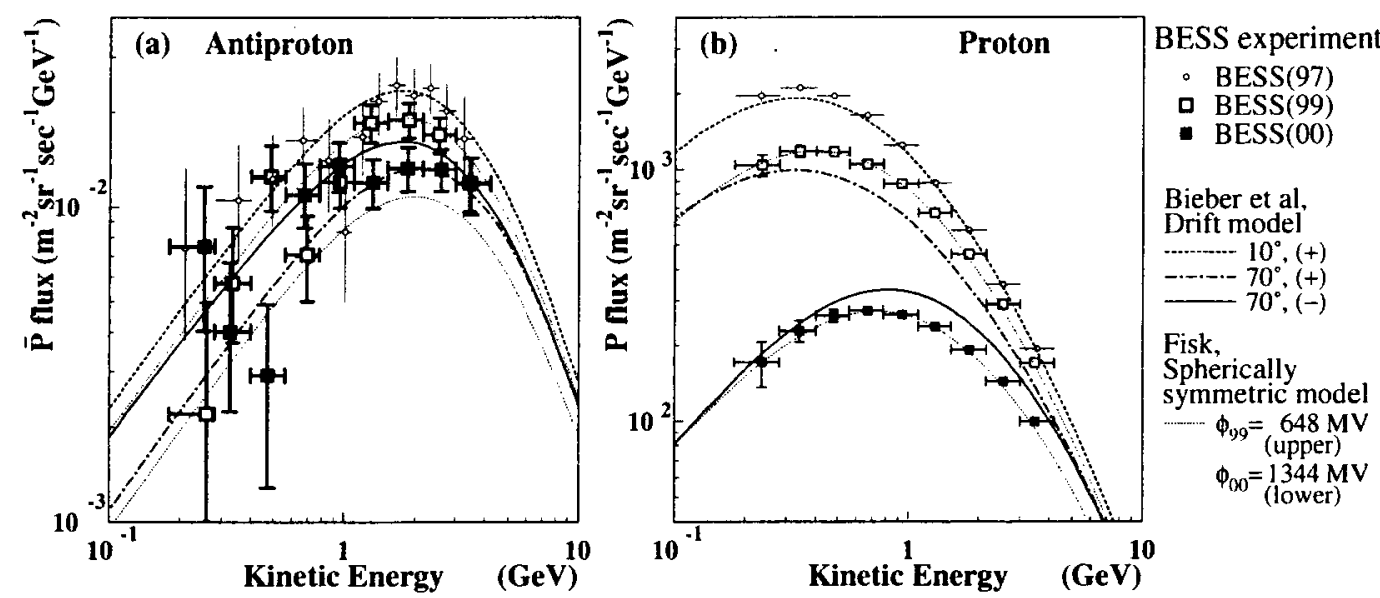

Fig. 4. The (a) antiproton and (b) proton fluxes measured by BESS in 1997, 1999, and 2000 compared to calculations of the effects of solar modulation.

Neutralinos are among the possible candidates for dark matter in the universe (Jungman, Kamionkowski, and Griest 1996, Bergstrom et al. 1999), and their annihilation could produce antiprotons at energies well below the kinematic threshold for the production of antiprotons as nuclear secondaries. This might yield a feature, a flattening of the spectrum below $\sim 500 \mathrm{MeV}$, that could be effectively used to search for dark matter signatures (Mitsui 1996; Welles, Moiseev, and Ormes 1999). Similar flattening of the low-energy antiproton spectrum could be caused by evaporation of primordial black holes (Maki et al. 1996). BESS results show some possible excess antiproton flux at low energy, spurring efforts toward lowering the energy limit of BESS and increasing its sensitivity with the BESS-Polar instrument.

\section{SOLAR MODULATION}

The major difficulty in the study of low-energy antiprotons is that they are strongly affected by solar modulation, which could mimic or obscure the signature of an exotic source. The study of solar modulation is very complex, and ideally involves long-term (extending over much of the solar cycle) measurements of all charged components of the cosmic radiation. It is probable that the modulation effect depends on the particle charge-sign and solar magnetic field polarity (Potgieter and Burger 1990, Tuska et al. 1991). Although the Sun has a complex magnetic field, the dipole term nearly always dominates in determining the magnetic field of the solar wind. At each sunspot maximum, the dipole reverses direction, leading to alternating magnetic polarity in successive solar cycles. The charge-sign dependence of solar modulation has been studied with electron and helium data (Clem et al. 2000). However, it is best studied using identical particles with opposite sign (e.g. positrons and electrons or antiprotons and protons). The antiproton to proton ratio is expected to strongly

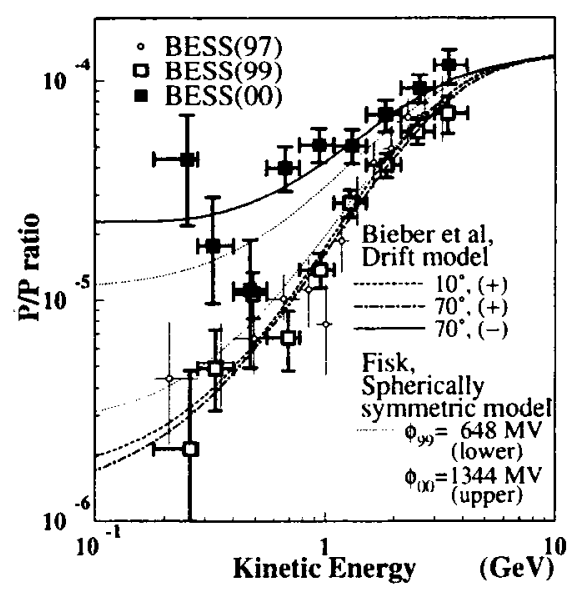

Fig. 5. The antiproton/proton ratio measured by BESS in 1997, 1999, and 2000 compared to model calculations. 
depend on the solar magnetic field polarity (Bieber et al. 1999), and is an ideal tool for the study of charge-signdependent solar modulation. BESS, with its yearly flights, provides a unique opportunity to track this dependence. Figure 4 (Asaoka et al. 2002) shows the absolute antiproton and proton fluxes from the BESS 1997, 1999, and 2000 flights. Figure 5 shows the resulting antiproton to proton ratio. There was a reversal of the solar magnetic field before the 2000 BESS flight. As indicated in Figure 4, increasing solar activity suppresses the relatively soft primary proton spectrum, while the spectrum of the secondary antiprotons is less strongly affected. The increase in the antiproton/proton ratio with increasing solar modulation results mainly from the relatively greater suppression of the primary protons and is shown by both spherically-symmetric and drift-model calculations (Bieber et al. 1999). However, the rapid increase in this ratio in the 2000 data is better reproduced by the drift model. It is very important to continue this investigation into and through solar maximum. Future BESS flights can provide the antiproton spectrum from $100 \mathrm{MeV}$ to $4.2 \mathrm{GeV}$ with increased sensitivity and statistics.

\section{SEARCH FOR ANTIHELIUM}

Beginning with the first BESS flight, a continuing search for antihelium has been carried out (Ormes et al. 1997, Saeki et al. 1998, Nozaki et al. 1999, Sasaki et al. 2002). The unambiguous detection of even a single antihelium would provide conclusive evidence for the existence of primordial antimatter. A comprehensive review of antimatter in the universe and the significance of the antihelium search was given by Streitmatter (1997). No antihelium candidate has been detected by BESS in $>6.6 \times 10^{6}$ observed helium events in a rigidity range of $1 \mathrm{GV}$ to $14 \mathrm{GV}$ accumulated in six flights. Figure 6 shows the upper limit of the antihelium/helium ratio derived from the BESS program in comparison with other experiments. The resultant upper limit of the antihelium/helium flux ratio at the top of the atmosphere has been decreased to 6.8 $\times 10^{-7}$ with a $95 \%$ confidence level under the assumption that antihelium and helium would have the same spectrum (Sasaki et al. 2002). The sensitivity of the antihelium search has been pushed nearly two orders of magnitude by the BESS experiment. The search for antihelium is to be extended to reach an upper limit $-10^{-7}$ in the future BESS long duration flights.

\section{THE BESS-POLAR INSTRUMENT}

BESS-Polar is an entirely new lightweight version of the instrument under construction for a polar long-duration balloon (LDB) flight in 2004. BESS-Polar is discussed in detail in Yamamoto et al. (2002) and Yoshida et al. (2002). The detector system consists of a JET central tracker, with TOF, ACC, and IDC for triggering and particle identification. The JET, IDC, and a middle TOF layer (MTOF), below the lower IDC, are

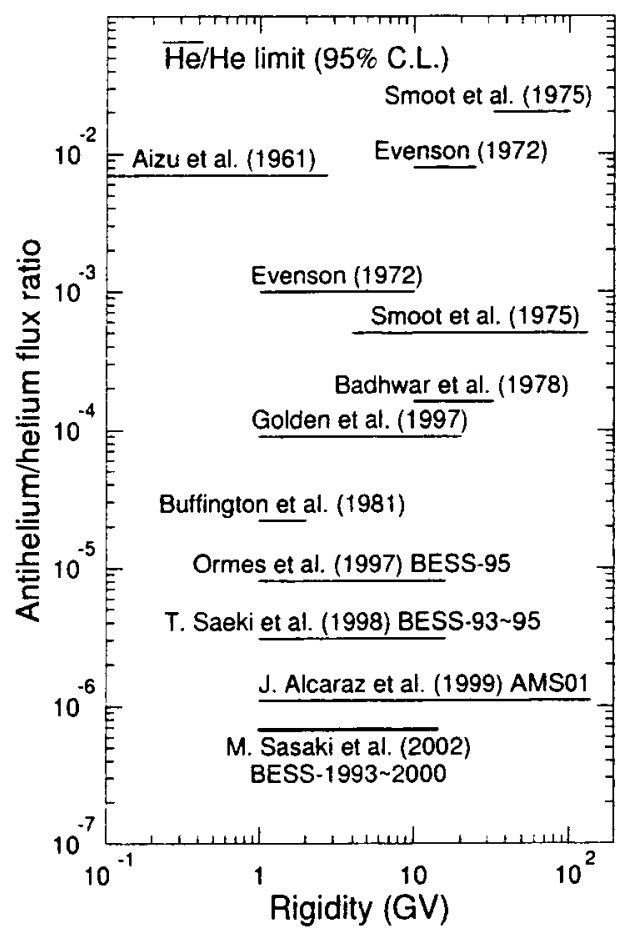

Fig. 6. The upper limit to the ratio of antihelium to helium obtained with BESS data through 2000 . placed inside the warm bore of the solenoid, which also acts as a pressure vessel. The outer TOF layers and ACC are in vacuum. A very low instrumental cut-off for antiprotons is achieved with a new thin-walled superconducting magnet, using a new high-strength aluminum-stabilized superconductor, and by eliminating the pressure vessel, thinning the TOF, adding the MTOF, and moving the ACC below the magnet. Low-energy particles only have to reach the MTOF to be detected, traversing $4.5 \mathrm{~g} / \mathrm{cm}^{2}$ of material, about four times less than in BESS-TeV. The lowest energy for antiproton detection is $\sim 0.1 \mathrm{GeV}(\sim 0.45$ $\mathrm{GeV} / \mathrm{c}$ ) at the top of the atmosphere (TOA). The cryogenic system of the magnet allows continuous operation for over 20 days and the instrument is powered by solar arrays. The compact spectrometer design has a geometrical acceptance of $0.3 \mathrm{~m}^{2} \mathrm{sr}$, matching the acceptance of the current BESS instrument. BESS-Polar is unique and complementary to two space experiments, PAMELA and AMS, to be carried out in the same time period (Yoshimura et al. 2001, Yoshida et al. 2002). BESS-Polar will conduct extremely sensitive measurements of lowenergy antiprotons, greatly improve the sensitivity of the antihelium search, and will have an improved ability to measure light cosmic-ray isotopes due to the increased transparency of the new magnet. 


\section{SUMMARY}

BESS is a sophisticated, very-large-geometry balloon instrument that is being fully exploited to understand the origin of cosmic-ray antiprotons, to probe galactic and heliospheric cosmic-ray transport processes, and to search for cosmic antimatter. BESS has measured the cosmic-ray antiproton spectrum between 0.18 and $4.20 \mathrm{GeV}$ in eight flights between 1993 and 2002. These data show that most of the antiprotons originate as secondary products of interactions of primary cosmic rays with the ISM. Below $1 \mathrm{GeV}$, however, BESS data suggest the presence of an additional source of antiprotons. BESS antiproton and proton spectra provide unique tools to study solar modulation. These data generally agree with a charge-sign-dependent drift model, and show the importance of a careful treatment of solar modulation for the interpretation of low-energy antiproton measurements. BESS has also carried out a sensitive search for antihelium and has set the most stringent current limit to the ratio of antihelium to helium. The antiproton measurements and antihelium search will be greatly extended by the BESSPolar instrument. Flying for long periods at the low polar geomagnetic cutoff, BESS-Polar will make unique contributions to the search for antimatter, dark matter, and primordial black holes.

\section{References}

Ajima, Y., K. Anraku, T. Haga, et al., Nucl. Instr. and Methods, A443, 71-100, 2000.

Anraku, K., R. Golden, M. Imori, et al, Adv. Space Res., 17, (9) 101, 1996.

Asaoka, Y., K. Abe, K. Yoshimura, et al., Nucl. Instr. and Methods, A416, 236-242, 1998.

Asaoka, Y., Y. Shikaze, K. Abe, et al., Phys. Rev. Lett. 88, 051101, 2002.

Basini, G., R. Bellotti, M.T. Brunetti, et al., Proc. 26 $6^{\text {th }}$ Int. Cosmic-Ray Conf. (Salt Lake City), 3, 77, 1999.

Beach, A.S., J.J. Beatty, A. Bhattacharya, et al., Phys. Rev. Lett., 87, 27101-1, 2001.

Bergstroem, L., J. Edsjo, and P. Ullio, Astrophys. J., 526, 215, 1999.

Bieber, J.W., R.A. Burger, R. Engel, et al., Phys. Rev. Lett. 83, 674, 1999.

Boezio M., P. Carlson, T. Francke, et al., Astrophys. J., 487, 415, 1997.

Boezio, M., V. Bonvicini, P. Schiavon, et al., Astrophys. J. 561, 787, 2001.

Clem, J.M., P. Evenson, D. Huber, et al., J. Geophys. Res. 105, (A10) 23099, 2000.

Jungman, G., M. Kamionkowski, and K. Griest, Phys. Rep., 267, 195, 1996

Maeno, T., S. Orito, H. Matsunaga, et al., Astroparticle Phys. 16, 121, 2001,

Maki, K., T. Mitsui, and S. Orito, Phys. Rev. Lett. 76, 3474, 1996.

Matsunaga, H., S. Orito, H. Matsumoto, et al., Phys. Rev. Lett. 81, 4052, 1998.

Mitchell, J. W., L.M. Barbier, et al., Phys. Rev. Lett, 76, 3057, 1996.

Mitsui, T., K Maki, and S Orito, Phys. Lett., B389, 169, 1996.

Moiseev, A.A., K. Yoshimura, I. Ueda, et al., Astrophys. J., 474, 479, 1997.

Nishimura, J., H. Matsunaga, M. Motoki, et al., Adv. Space Res. 19, (5) 771, 1997.

Nozaki, M.. M. Sasaki, T. Saeki, et al., Proc. 26 ${ }^{\text {th }}$ Int. Cosmic-Ray Conf. (Salt Lake City), 3, 85, 1999.

Orito, S., Proc. Astromag. Workshop, KEK Report 87-19, 111, 1987.

Orito, S., T. Maeno, H. Matsunaga, et al., Phys. Rev. Lett, 84, 1078, 2000.

Ormes, J.F., A.A. Moiseev, T. Saeki, et al., Astrophys. J., 482, L187, 1997.

Potgieter, M.S., and R.A. Burger, Astron. Astrophys., 233, 598, 1990.

Saeki, T., K. Anraku, S. Orito, et al., Phys. Lett. B422, 319, 1998.

Sanuki, T., M. Motoki, H. Matsunaga, et al., Astrophys. J., 545, 1135, 2000.

Sanuki, T., Y. Yamamoto, M. Motoki, et al., Proc. $27^{\text {th }}$ Int. Cosmic-Ray Conf. (Hamburg), 950, 2001 a.

Sanuki, T., H. Matsumoto, M. Nozaki, et al., Adv. Space Res., 27, (4) 761, 2001b

Sasaki, M., H. Matsumoto, M. Nozaki, et al., Proc. SpacePart 2002 Conf. (Elba), submitted to Nucl. Phys. B, 2002.

Shikaze, Y., S. Orito, T. Mitsui, et al., Nucl. Instr. and Methods, A455, 596, 2000.

Streitmatter, R.E., Il Nuovo Cimento, 19, 835, 1997.

Tuska, E., P. Evenson, and P. Meyer, Astrophys. J., 373, L27, 1991.

Wells, J., A. Moiseev, and J.F. Ormes, Astrophys. J., 518, 570, 1999.

Yamamoto, A., K. Anraku, R. Golden, et al., Adv. Space Res., 14, (2) 75, 1994.

Yamamoto, A., S. Orito, J. Ormes, et al., Proc. $21^{\text {st }}$ Int. Symp. on Space Tech. and Sci. (Omiya), 1578, 1998.

Yamamoto, A., K. Abe, K. Anraku, et al., Adv. Space Res., 30, (5) 1253, 2002.

Yoshịda, T, A. Yamamoto, J. Mitchell, et al., submitted to Adv. Space Res., 2002.

Yoshimura, K., S. Orito, I. Ueda, et al., Phys. Rev. Lett., 75, 3792, 1995.

Yoshimura, K., Adv. Space Res., 27, (4) 693, 2001.

Email of John W. Mitchell: John.W.Mitchell@nasa.gov

; accepted 